\title{
An Architecture for Automatic Deployment of Brown Dog Services At Scale into Diverse Computing Infrastructures
}

\author{
Smruti Padhy, Jay Alameda, Rob Kooper, Rui Liu, Sandeep Puthanveetil Satheesan, \\ Inna Zharnitsky, Gregory Jansen, Michael C. Dietze, Praveen Kumar, Jong Lee, \\ Richard Marciano, Luigi Marini, Barbara Minsker, Chris Navarro, Marcus Slavenas, \\ William Sullivan, Kenton McHenry \\ National Center for Supercomputing Applications \\ University of Illinois at Urbana-Champaign \\ \{spadhy, alameda, mchenry\}@illinois.edu
}

\begin{abstract}
Brown Dog is an extensible data cyberinfrastructure, that provides a set of extensible and distributed data conversion and metadata extraction services to enable access and search within unstructured, un-curated and inaccessible research data across different domains of sciences and social science, which ultimately aids in supporting reproducibility of results. We envision that Brown Dog, as a data cyberinfrastructure, is an essential service in a comprehensive cyberinfrastructure which includes data services, high performance computing services and more that would enable scholarly research in a variety of disciplines that today is not yet possible. Brown Dog focuses on four initial use cases, specifically, addressing the conversion and extraction needs in the research areas of ecology, civil and environmental engineering, library and information science, and use by the general public. In this paper, we describe an architecture that supports contribution of data transformation tools from users, and automatic deployment of the tools as Brown Dog services in diverse infrastructures such as cloud or high performance computing (HPC) based on user demands and load on the system. We also present results validating the performance of the initial implementation of Brown Dog.
\end{abstract}

\section{CCS Concepts}

-Information systems $\rightarrow$ Extraction, transformation and loading; Computing platforms; •Networks $\rightarrow$ Cloud computing; •Software and its engineering $\rightarrow$ Software as a service orchestration system;

\section{Keywords}

Elasticity; HPC; Cloud; Data conversion; Metadata extraction; Data cyberinfrastructure; Digital preservation; $\mathrm{Au}-$ tocuration; Ecology; Civil and Environmental Engineering; Library and Information Science

Permission to make digital or hard copies of all or part of this work for personal or classroom use is granted without fee provided that copies are not made or distributed for profit or commercial advantage and that copies bear this notice and the full citation on the first page. Copyrights for components of this work owned by others than ACM must be honored. Abstracting with credit is permitted. To copy otherwise, or republish, to post on servers or to redistribute to lists, requires prior specific permission and/or a fee. Request permissions from permissions@ acm.org.

XSEDE16, July 17-21, 2016, ,

(C) 2016 ACM. ISBN 978-1-4503-4755-6/16/07 . \$ $\$ 15.00$

DOI: http://dx.doi.org/10.1145/2949550.2949647

\section{INTRODUCTION}

Research across different scientific disciplines today often involve in the development of software to access raw data, process and analyze the data, and produce new scientific results. Much of these data generated or used in the process are unstructured and uncurated, and come in a variety of formats. With fast technology changes and with little provision for data management in the grants, these data and software are less managed and become inaccessible over time thus hindering the reproducibility of the scientific results and the future research. There have been several research efforts towards preservation and reproducibility of the scientific results, e.g. building scientific workflow system towards preserving the execution provenance trail $[1,9]$, providing repositories for domain specific scientific code/tools [4, 7], developing user friendly content management systems [10], developing frameworks for file format conversions and dealing with information loss $[11,17]$, building test suites [5], and efforts involving use of computer vision [16] and natural language processing [3].

We focus on another research direction of the problemGiven a large collection of unstructured scientific data that lacks metadata describing its content, how to make it more managed, searchable, and to make it available in an accessible file format. There are also efforts that tries to address this problem by allowing users to curate their data or do some file format conversion $[13,14]$. There also exists several software/tools/frameworks across different scientific domain that provide metadata extraction and file format conversion capabilities. However, there is a need of a framework that could orchestrate and leverage these capabilities of the existing tools/software/frameworks/service across different disciplines and provide them as as services. Towards this, the NSF funded Brown Dog project ${ }^{1}$, which is part of the DataNet/DIBBS program, has developed the Brown Dog (BD) framework [15] that provides a set of highly extensible and distributed services, the Data Access Proxy (DAP) and the Data Tilling Service (DTS) for data format conversion and metadata extraction from data content, respectively. DAP and DTS can both leverage any existing software's conversion/extraction capabilities or integrate new software for the needed conversion and extraction purposes. The core services are accessible through a set of consistent and programmable REST APIs.

\footnotetext{
${ }^{1}$ browndog.ncsa.illinois.edu
} 
Thus, BD provides researchers with the ability to use existing tools or to write a new tool to unlock inaccessible data, curate data and enable indexing of unstructured data collection. After writing a new data conversion/extraction tool, as next step, a researcher should be able to submit the new tool to BD which later will be deployed as part of BD services. To this extent, a new component, Tools Catalog was added to BD. In [15], the authors introduced the idea of Tools Catalog with not much details on the design. In this paper, we describe the design and latest capabilities of the Tools Catalog.

The BD services use a distributed messaging bus, specifically RabbitMQ ${ }^{2}$, with multiple queues, for placing conversion/extraction requests received from clients or users to the respective queues for conversion/extraction tools. Data conversion tools are known as converters and reside in Software Servers (SS) [12] that expose their capabilities as services. Similarly, metadata extraction tools are known as extractors and exist as extraction services. Both SS and extractors can be deployed in distributed environments such as cloud or high performance computing (HPC) resource.

To deal with dynamic increase or decrease in user demand and load in the BD system, several instances of an extractor/SS should be deployed dynamically. To address scalabililty issues and to dynamically grow/shrink extractors/SS instances, Elasticity Module (EM) has been added to the Brown Dog framework. It monitors the queue length for specific extractor/SS and auto scales up/down instances when the queue length exceeds/falls below some threshold. In [15], the design of EM was discussed and experimental evaluation results were provided. However, to meet the needs of large data with large computational demands, as well as the desire for lower virtualization overhead, the design of EM has been improved since then so that it could use docker as another level of virtualization within a VM in an OpenStack Cloud and new options have been added to choose the computing infrastructure for deployment. A user or an administrator would decide to deploy these instances in cloud or on HPC depending on the computational needs to process the data. We discuss EM design changes and the new options added to it. Additionally, we discuss necessary modifications to extractors to allow them to be deployed to the HPC environment.

From a BD user perspective, an ideal case would be to write a new extractor/conversion tool, submit it to Tools Catalog and see the tool easily being deployed within BD services and available for use and sharing as soon as possible. From BD admin perspective, ideally, the new tools should be rapidly tested for validity and correct input/output and then EM should deploy the new tool with minimal admin effort. In this paper, we provide an architecture that integrates Tools Catalog and EM module and eases the workflow from submission of a new tool till deployment thereby saving lots of manual effort of admin and timely deployment of the tool for the user.

The core contribution of this paper is an architecture that supports contribution of data transformation tools from users, and automatic deployment of the tools as Brown Dog services in diverse infrastructures such as cloud or high performance computing (HPC) based on user demands and load on the system. The concepts involved in the design of EM,

\footnotetext{
${ }^{2}$ www.rabbitmq.com
}

HPC deployment and integration architecture are generic enough and can be used in the design of automated deployment infrastructure in a distributed environment. The EM and the Tools Catalog are independent open source modules which can be used with minimal modification in conjunction with other distributed services that uses RabbitMQ as messaging technology and an OpenStack Cloud infrastructure.

We will use the terms- extractors/SS and BD services - interchangeably throughout this paper. We organize the paper as follows: Section 2 explains the use cases of $\mathrm{BD}$ and their conversion/extraction needs; Section 3 describes an architecture for automatic deployment of BD services in cloud infrastructures while Section 4 describes deployment of BD services in HPC resources; Section 5 provides experimental validation of the architecture explained in earlier sections; and Section 6 discusses the status and the future work.

\section{USE CASES}

BD focuses on four initial use cases, specifically, addressing the conversion and extraction needs in the area of research in ecology, civil and environmental engineering, library and information science, and general public use.

In ecological science, researchers often simulate, study and predict land surface carbon sinks over time in different geographical areas. They use a variety of models for their studies which requires data to be in specific format. An integrated ecological bioinformatics toolbox, Predictive Ecosystem Analyzer (PEcAn) [2], uses BD conversion services to provide data from different sources (e.g. Ameriflux ${ }^{3}$, $\mathrm{NARR}^{4}$ ) to model specific formats for their study.

$\mathrm{BD}$ services are also being tested and applied to archival collections [15], specifically CI-BER collection hosted at the UMD Digital Curation Innovation Center. CI-BER consists of 72 million files with a total of 52 terabytes. These files range from a variety of file formats, datasets and variety of records. This use case is developing archival specific extractors and converters, and building a CI-BER data infrastructure for archival format conversions, curations and analyzing the extracted data contents for valuable information.

$\mathrm{BD}$ is also providing support towards of data transformation needs in green infrastructure and civil engineering research specifically, obtaining greenness index and calculating human preference index from a collection of images, sentiments analysis on collection of input text for human preference modeling, extracting floodplain surfaces from lidar data for hydrogeomorphological studies, etc.

The general public also faces challenges in their day to day life with format conversions (e.g. ps to pdf or doc to pdf) and managing their unstructured data such as documents formats, images, videos (e.g. extract graph/table from a pdf when no source is there, managing files based on some features such as faces, eyes, etc). Brown Dog addresses these conversion and extraction needs.

In all of these use cases, the SS and extractors developed are designed to be deployed in cloud. However, there are several other use cases that drive the design of BD framework to support deployment on HPC. For example, the Video Analysis Tableau (VAT) project (formerly known as Large Scale Video Analytics (LSVA)) [8] using XSEDE HPC resource [6], tries to solve the problem of analyzing large collections

\footnotetext{
${ }^{3}$ ameriflux.lbl.gov

${ }^{4}$ www.esrl.noaa.gov/psd/data/gridded/data.narr.html
} 
of videos from archives, which is computationally complex and has large data transformation requirements.

Another example is the TERRA-REF project ${ }^{5}$ (Transportation Energy Resources from Renewable Agriculture Phenotyping Reference Platform). To accelerate energy crop development for the production of renewable transportation fuels from biomass, the research focus has been on a breeding program which depends on speed at which phenotypes can be measured and can be predicted. As part of TERRA-REF project, the goal is to instrument a field with a gantry system that will monitor the growth of the plants (Sorghum) and collect data. The gantry system will scan the field at high resolution with many different sensors (camera, infrared, hyperspectral, 3D sensors, etc), as well as measure the environmental conditions such as temperature, humidity, etc. These measurements from sensors will generate large volume of data that needs to be processed to obtain plant phenotypes (traits).

\section{DEPLOYMENT OF BD SERVICES IN CLOUD INFRASTRUCTURES}

\subsection{Elasticity}

The two BD core services, DAP and DTS, need to handle dynamic user demands for conversions and extractions, respectively, and require to autoscale based on the demands and load on the system. To this extent, an Elasticity Module (EM) was designed and implemented within BD framework. The EM is a stand-alone Python program that monitors the RabbitMQ queues and takes scaling actions based on some conditions, for instance when the SS/extractor instance queue lengths exceed certain thresholds, EM uses a cloud API to automatically deploy a new Virtual Machine $(\mathrm{VM})$ /resume a suspended VM with required number of respective extractor/SS instances or deploy instances on an existing Virtual Machine based on the load on the VM. The NCSA Nebula, an OpenStack Cloud infrastructure, was used for the deployment of instances of SS/extractors. In [15], an initial EM's design, algorithms and implementation are described.

A VM deployed in the cloud to provide BD services, would consist of instances of either SS or extractors that require same execution environments and third-party software. Though such design addressed the heterogeneous requirements of OSes (e.g. different versions of Windows and Linux) and third-party software for SS/extractors (e.g. OpenCV ${ }^{6}$ (Open source Computer Vision), Tesseract, imagemagick, MATLAB, ArcGIS, etc.), there are some overheads associated to it such as large resource usage to provide execution environment isolation for each type of SS/extractor, and large startup time of each VM. Thus, besides the capability of using Cloud VM images to deploy extractors/SS, we extended the EM to support deploying using Docker container images. Docker $^{7}$ implements a high-level API to provide lightweight software containers that run processes in isolation on a Linux kernel. It allows independent containers to run within a single Linux instance, avoiding the overhead of starting and maintaining virtual machines.

\footnotetext{
${ }^{5}$ terraref.org

${ }^{6}$ opencv.org

${ }^{7}$ www.docker.com
}

The EM now supports deployment methods using both VMs and Docker container images simultaneously. For simplicity, the support is one deployment method per tool type, i.e., one tool type uses exactly one deployment method, but different tool types can use different deployment methods. Each Docker container image contains either an extractor or a SS. We currently use the Docker $\mathrm{Hub}^{8}$ for Docker image storage. Docker-VMs are VMs that use a manually created, updated Linux VM image with Docker engine installed to manage Docker containers. Several extractors/SS Docker containers can be deployed in separate or same Docker-VMs with no restriction to their type or the same execution environment requirement seen with pure VMs. Thus, a DockerVM has another layer of virtualization to a VM and provides execution environment isolation for extractors/SS. DockerVMs and their corresponding containers' life cycles are managed by the EM. The Docker daemon in a Docker-VM is used to watch and to restart the containers, so they remain up if occasionally crashed. Similar to the support for VM images, both scaling up and scaling down are supported for Docker containers.

The scaling up and scaling down algorithms for the Docker deployment method are similar to those for VM images described in [15]. The scale-up/down algorithm takes action at two levels- service/container-level and VM/Docker-VM level based on queue lengths and VM's CPU-load average.

However, we need to handle differently for the deployment method of VM images and docker containers, depending on which is used for a particular extractor/SS type.The main differences between the two deployment methods are: (i) for VM images, an OS system service is used to start or stop an extractor/converter type, while for Docker containers, Docker commands are used to start or stop/remove a container, (ii) when starting a new Docker VM, EM needs to wait for the Docker VM to finish start up, then run a Docker command to start a container; while in the VM image case, when a VM instance is started, the extractor/converter will automatically start since it is configured as an OS service, so EM does not need to wait for starting the VM to finish, (iii) when a Docker-VM is started, no container runs on it, so RabbitMQ will not report it as an available VM to add extractors/converters. The EM needs to keep track of such empty Docker VM as an available VM.

From architectural perspective, the EM module has five functional blocks: 1) Cloud infrastructure interaction, using the OpenStack API to start/ resume/suspend a VM/DockerVM; 2) Job monitoring - monitoring RabbitMQ queue lengths and idle times; 3) VM/ Docker-VM interactions - executing commands on VMs/ Docker-VMs to start/stop tool instances/containers; 4) Scaling actions- scaling the system up/down using the configuration file and other components, and 5) Logging - writing queue statistics and elasticity actions into a database, for analysis and visualization.

\subsection{Tools Catalog}

Tools Catalog (TC), another building block of BD, is a web application that provides a platform for users to add information on new tools they build or any existing tools, share the tools with the community, and get credit and citations for their work. These tools are the original software developed by a researcher or third party software, with conversion and extraction capabilities, e.g., OpenCV, Tesser-

\footnotetext{
${ }^{8}$ hub.docker.com
} 
act $^{9}$, NLP Toolkit ${ }^{10}$, imagemagick ${ }^{11}$. The TC also allows users to upload BD tools such as extractors and converters that wrap the original/third-party software to provide specific conversion/extraction. The BD tools, alternatively known as BD scripts, are created with the lightweight wrapper scripts or using a library provided by $\mathrm{BD}$ to convert a tool to deployable BD tool [15]. For example, a BD tool could be an extractor that uses OpenCV to extract human faces from an image, or a converter that uses imagemagick to convert an input file of jpg format to png format. Other functionalities of TC include ability to browse, search and download BD tools, and allow administrative users to deploy new BD tools in a deployment platform such as a cloud and start/stop tool instances. Conceptually, TC has similarities with Apple's AppStore, nanoHUB's Tool Sharing, and Galaxy Toolshed, while it has features specific for Brown Dog users. The vision is that in the future, it can be extended to allow users to ask questions and get answers regarding the tools, provide reviews to tool authors, and help create a sense of community. The TC's functionalities are accessible through web user interface which has been made simple and easy to use.

Based on Apple AppStore's two-level model approach, TC is designed to have two levels - the tool, and the Brown Dog tool/script level. At the tool level, while adding a tool a user needs to provide the information about the tool such as title, a short/detailed description, reference to the tool as URL, citation information in terms of reference to published paper or software to get proper acknowledgments/credits, any video on the tool and the tool type. The tool is not stored in the TC but a reference to it so that a user uses the reference for subsequent tool download. At the Brown Dog tool/script level, a user is presented with options to upload $\mathrm{BD}$ tool/script file, docker file with information on deployment of $\mathrm{BD}$ tool on a docker container, a sample input file and a sample output file. In addition to the above information on tools/BD scripts, a tool within TC has several properties such as number of downloads, creation date, update date, script level number, what's new, and an icon. Figure 2 shows a screenshot of the TC browse webpage with a list of available tools information and shows option to search and contribute.

The creation, and managing of tools and their corresponding BD tools/scripts require a careful design of the workflow. The workflow is the procedure of how a user creates, uploads, submits a tool for admin approval, retires a tool, how an admin approves or rejects a tool for publishing, and revokes and re-approves a tool - in essence, defining the life cycle of a tool.The TC follows a Tool Status Workflow as shown in Figure 1. The TC is implemented as a web application, as much of the operation involves user interactions. To persist and preserve the data pertinent to tool (both the actual tool and the BD tool/script), a database is used. We chose a NoSQL database, MongoDB, as it provides the flexibility and ease of adding more fields for a tool as the TC evolves, and provides sharding across machines out of the box to scale TC horizontally.

We also incorporate a few admin capabilities, such as review, revoke and re-approve tools/BD tools (scripts), browse

\footnotetext{
${ }^{9}$ github.com/tesseract-ocr/tesseract

${ }^{10}$ www.nltk.org

${ }^{11}$ www.imagemagick.org
}

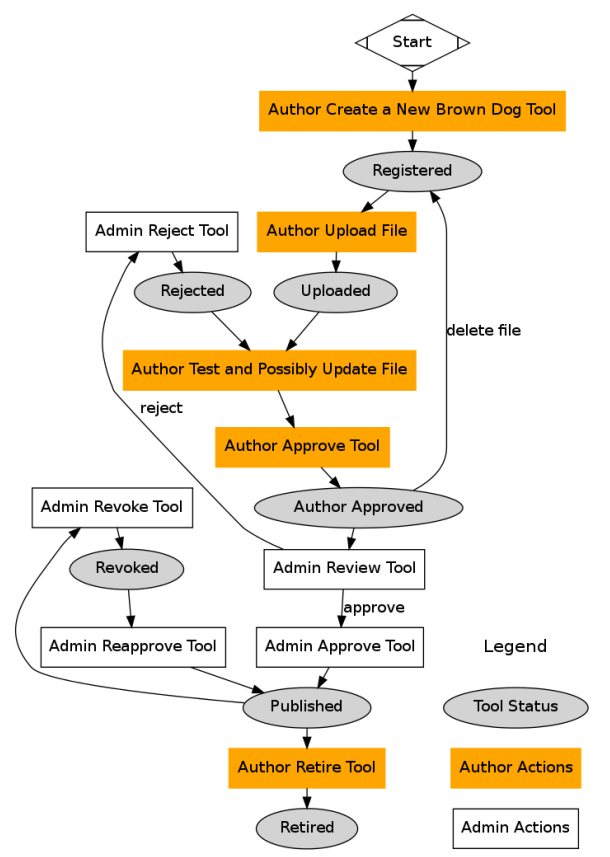

Figure 1: Tool status workflow in Tools Catalog.

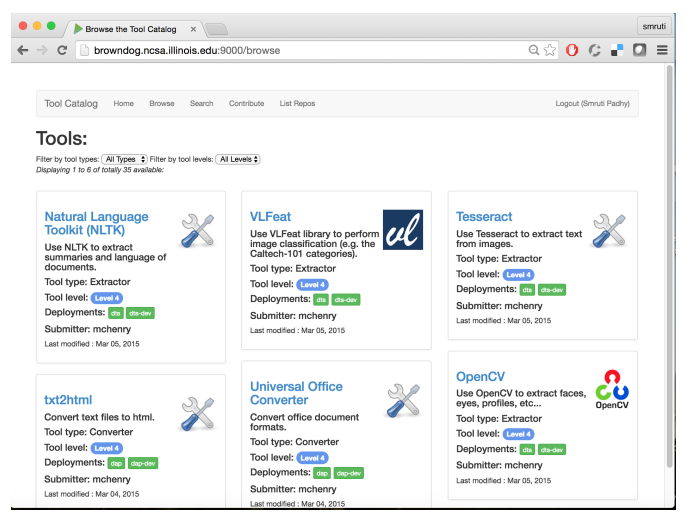

Figure 2: Tools Catalog's browse webpage showing the list of available tools and their information at a glance.

all tools including these that do not have a BD tool/script associated with it in the Published status. Indexing is done for the tool and BD tool/script fields to enable searching. TC also provides admin option to choose BD tools/scripts, number of instances of it for deployment and deployment methods such as Cloud.

\subsection{Integration of Tools Catalog with Elastic- ity Module}

In the initial design, TC and EM are two independent functional modules in the framework. That is, when a new tool is added to the TC, the admin verifies the submitted tool and approves/rejects it. On approval, the tool is visible to the community and could be shared/downloaded from TC. For deployment, the admin would download the file and manually deploy it within a computing infrastructure and then EM would watch it for scaling. To ease this process of deployment, we integrated TC and EM so that they can communicate to automatically deploy the tool in timely manner requiring minimal admin effort through a web in- 
terface. Additionally, the same web interface provides the ability to stop a running tool/BD script. Currently, deployment using cloud infrastructure supports using both Docker images and VM images. We are also working towards incorporating other computing infrastructure such as HPC resources.

Since the Brown Dog services and EM uses a RabbitMQ message bus for messaging and job monitoring, we use it for TC-EM communication as well. We added a listener thread to EM to listen to any deployment request from TC via RabbitMQ request queue. The JavaScript Object Notation (JSON) format is used as the exchange data format, since it is well supported in Python (EM) and Scala (TC).

The workflow of the EM-TC integration is:

1. An admin user submits a deployment/stop request using TC into a request queue in RabbitMQ, specifying the tool/script, cloud, docker image and other details (such as the minimum/maximum number of tool instances to be deployed);

2. The listener thread in EM processes and validates the request;

3. The listener thread either rejects it or if validated, generates a new configuration file;

4. The listener thread sends the response back to TC, in a response queue in RabbitMQ;

5. The TC processes the response, and stores it for future monitoring and analysis;

6. The EM main thread checks the configuration file in each iteration, and reloads it if it changed;

7. The EM main thread deploys the tool in the cloud or stops it according to the new configuration file.

This design has the following advantages: i) only on-demand traffic is generated; ii) there is no need to create another web service, thus simplifies the design; iii) in EM, we added a listener thread to handle TC/EM interaction, so integration with TC is de-coupled from EM's main thread. This looselycoupled modular approach allows TC, EM listener thread, and EM main thread to be developed, debugged and tested separately, as long as they communicate using an agreedupon interface.

\section{DEPLOYMENT OF BD SERVICES ON HPC RESOURCE}

The data transformation steps within extractors/SS may take a small or large time based on specific data type, format, size or any existing software used under the hood. For example, detecting and extracting faces from a jpeg image content may take few seconds while extracting shots/frame information from a video may take several minutes depending on its size.

For instance, according to YouTube statistics ${ }^{12}$, every minute, more than 300 hours of video gets uploaded into it and YouTube is only one among the many video hosting services. There are also many archives that are not accessible to public. The amount of total video data in the digital and

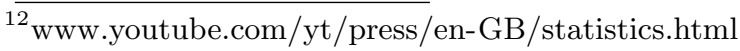

non-digital world is gargantuan. It is not humanly possible to analyze or for that manner even view all these video files. A video of resolution $640 \times 480$ and duration 60 minutes with frame rate 30 fps contains 108,000 frames $(30 \times 60 \times$ 60 ). A dataset containing 20 similar videos will have about 2 million frame images that amounts to roughly 660 billion pixels that needs to be processed. This clearly illustrates the the computational complexity and data requirements of the problem. The VAT project tries to solve this problem by building different extractors, namely, video preview, and detection, shot indexing, and movie slice visualization [8], supported with use of high performance computing (HPC).

Another BD HPC driver is providing support for the computational and data transformation needs of of the TERRAREF which aims to build high-throughput phenotyping system to accelerate plant breeding. Currently, TERRA-REF is expected to receive around $5 \mathrm{~TB}$ of data per day. All this data collected will need to be processed as quickly as possible (within 24 hours). The computational pipeline that is being developed as part of the TERRA-REF project is not a static pipeline but will grow over time. Some of the tasks that are to be performed can be done quickly, while others will take significantly longer requiring HPC resources. Finally, the data that is received will come from many different sensors, where each sensor has its own compute pipeline.

So far, the focus of EM has been mostly on using cloud infrastructure for deployment of extractors/SS that are designed as cloud based services. However, with large data in some of the use cases and requiring large computing resources, there is a need within BD to provide support for the use of HPC resources for its services. The vision is to provide options within TC for admin or user to chose HPC for deployment of tools with high computational requirements and also add an option in EM to automatically deploy extractors on $\mathrm{HPC}$ when the queue lengths becomes very very large. In this paper, we present a design as how extractors can be deployed on HPC and scalability achieved in the process. We also describe a library specifically built for user to write extractor designed for HPC.

There are two approaches to run extractors on HPC resources: i) Create and run multiple extractor instances on HPC compute nodes as a single job, ideally using EM for automatic submission of the job; ii) Run a single instance of extractor designed for HPC and submission of a file or a combination of files for processing becomes a single job. In subsequent subsections, we describe each of these approaches.

\subsection{Multiple extractor instances deployment on HPC}

As a first approach to run extractors on HPC and to achieve scalability, a single job consisting of multiple instances of an extractor is submitted to HPC. In the meantime, extraction requests from $\mathrm{BD}$ users through $\mathrm{BD}$ clients get queued up at the RabbitMQ extractor queue. When the job starts, it launches multiple extractors using the GNU parallel tools with each extractor being run on each core. Each extractor listens to the same queue (owing to the same extractor) and picks up a request in a round-robin fashion from the queue. Thus we are able to process multiple files in parallel as part of a single HPC job. However, parallelism achieved through the number of extractor instances does depend on the number of available cores and the RAM that are requested when submitting the job. 
To minimize waste of resources, we try to strike a balance between the number of requests/files that needs to be processed, number of extractor instances in a job, and the requested wall time for the job. Hence, this approach is best suited in situations where we have at least a rough estimate of the number and size of files that needs to be processing. If this information is not available, the system can submit a job that starts the extractors and can kill the job when no requests are there. Then it will wait for more requests before submitting another job to HPC.

Although HPC jobs required specification of wall clock limits, our approach using RabbitMQ monitors the extractors and will requeue the incomplete requests at the termination of the batch job due to wall clock limits. This will also allow us to use the jobs as filler jobs. For example when scheduling jobs in HPC centers sometimes nodes could be sitting idle. Since our jobs can be terminated at any point (without losing any information), they can be scheduled to fill those gaps. This will ensure maximum utilization of the HPC resources.

\subsection{Extractor Design for HPC}

A second approach to run an extractor on HPC is to design extractor specifically for HPC.

Clowder $[10,14]$ is an open source web based content management system that enables extraction of metadata and signatures from data contents, generation of previews, sections, tagging and social curation via a set of extractors. BD extraction service, i.e. DTS, is built on top of Clowder (See [15] for detailed architecture of BD). In the initial extractor design, an extractor connects to the Rabbitmq server, listens for any new extraction request message, processes the file for which the request is made, returns the results (e.g., metadata, signature, tags, sections, preview) directly to Clowder using Clowder API, and sends status messages of the file processing to Clowder via the Rabbitmq message bus. To make writing of an extractor easy and simplified specifically in Python, we provided a library called pyClowder. pyClowder ${ }^{13}$ is a python package that contains methods for communication with RabbitMQ and methods that wrap Clowder API for upload/download of files/metadata/sections/previews to/from Clowder. So, with pyClowder in place, a developer writing an extractor has to implement just the process_file method that would extract information from the file customized to their application, and all communications to Rabbitmq and to Clowder are taken care by pyClowder library. Extractors designed in this way are easily deployable in a cloud infrastructure. However, to deploy an extractor in HPC requires extra effort and some design changes. This is because the steps needed to run an extractor on one HPC resource could be different from another because of differences in environments. Towards this, we provide a mechanism for easy extractor deployment on an HPC environment. To help a developer concentrate on writing the process_file method for an extractor for HPC environment, we extended the pyClowder library with HPC related methods. Design modifications are made such that the current extractors can be reused in HPC environment with minimal changes.

We describe here two pieces of code, which we developed, that reduce the effort needed run extractors on HPC re-

\footnotetext{
${ }^{13}$ opensource.ncsa.illinois.edu/bitbucket/projects/CATS/ repos/pyclowder
}

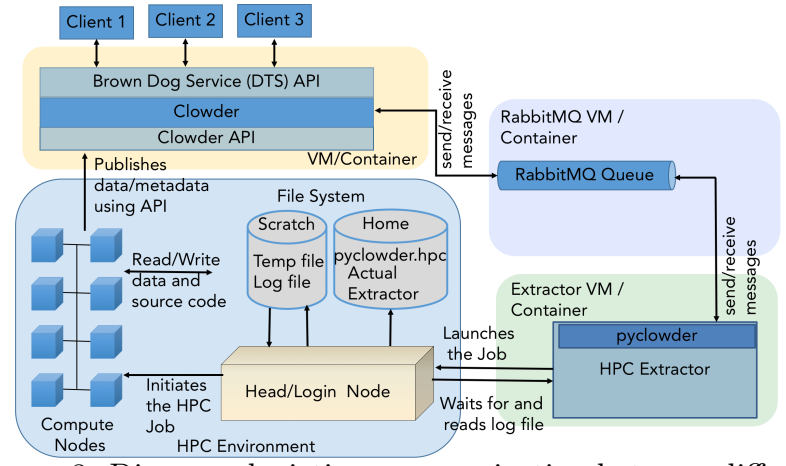

Figure 3: Diagram depicting communication between different Brown Dog components while using HPC resources.

sources. They work in tandem to submit jobs to a HPC resource and communicate status back to Brown Dog Services via RabbitMQ-Clowder.

The first one is a generic HPC extractor ${ }^{14}$ that does all required communications with Clowder via RabbitMQ, launches job on HPC and sends the status back to Clowder via the message bus. By generic we mean that once completely developed, the HPC extractor should be able to submit jobs to commonly used HPC environments, e.g. XSEDE resources. The second one is an HPC module called pyclowder.hpc added to the existing pyClowder library that extends pyClowder library with HPC specific methods. The processing of file is carried by an actual (core) extractor. An actual extractor is a program that processes the file of specific MIME type, extracts features/metadata out of it, and sends the extracted metadata to Clowder for BD service use. The actual extractor implements the process_file method and uses pyclowder.hpc for communications with Clowder and within HPC environment. It should be installed in the HPC environment where we would like to process the files. Figure 3 shows the communication flow between HPC extractor, Actual Extractor, and Clowder for deployment of an extractor in HPC environment.

The HPC extractor is a highly configurable python program that is written using pyClowder package. It listens to a RabbitMQ queue for specific file MIME types, for extraction requests. It picks up any request available on the queue, saves all information received in the request in a dictionary variable It also downloads the file that needs to be processed and save the new downloaded file path. Note that HPC extractor does not reside in the HPC resource but rather on an arbitrary container, and it does not process the file. Thus, the HPC extractor acts as broker that does the setup/configuration and required preprocessing of information, which is received from RabbitMQ, to submit a job to HPC, submits the job to HPC job scheduler, keep tracks of the status of the job when it is launched and sends status messages via message bus and an acknowledgment to BD service through Rabbitmq when the job is done.

The HPC extractor opens a SSH connection with the HPC resource, creates a temporary directory within the scratch directory and copies dowloaded file to that temp. directory. It also creates a log file where the status updates from the actual extractor will be written and updates all parameters

${ }_{14}$ opensource.ncsa.illinois.edu/bitbucket/projects/CATS/ repos/extractors-hpc 
such as file path and log file path in the parameters data structure, serializes the data structure to a file, called as pickle file and sends the pickle file to the HPC head node. It also sends the job script to the HPC head node to be run on the head node itself. This job script depends on the job scheduler that is being used by the HPC resource. Currently, we are supporting only TORQUE based systems while it is our aim to support most of the commonly used job schedulers in the future. The HPC extractor remotely runs the job script with two command line arguments - the actual extractor (core) program file path and pickle file path, and submits a new job to the HPC queue where it waits till it is picked up by the job scheduler and execution of the job begins.

When the job starts running, it starts the actual extractor on a compute node. The actual extractor is written using pyclowder.hpc package. Note that the pyclowder.hpc package contains methods related to HPC functionalities, specifically, one method that prepares a file to be processed within the HPC environment and another method to write status messages to a log file in the working directory. During processing of the file, generated metadata are sent to the Clowder and are saved in the database. The status messages generated by the actual extractor gets written to the log file whose path was passed in as a parameter. The HPC extractor remotely parses to this log file and starts sending these status messages back to Clowder as status messages are written by the actual extractor.

\section{EVALUATION}

\subsection{Elasticity}

In [15], we presented the evaluation results for the VM images deployment method. In this paper, we provide experimental evaluation of the Elasticity Module for the newly added support for the docker deployment method. The experiments are designed to validate the effectiveness of the EM module with respect to autoscaling using Docker and get more understanding and insights of the system. Our experimental setup consists of i) 5 extractors, dockerized and uploaded to DockerHub, ii) one Docker Openstack VM image, iii) single instance of DTS, iv) an OpenStack cloud, v) 60 test images, and vi) a set of defined elasticity parameters. The detail setup information and parameters are shown in Table 1.

We used NCSA Nebula OpenStack Cloud platform for tests. In each test, we uploaded 20 iterations of the 60 images for metadata extraction using the DTS REST API. Each of the five extractors processed all 1200 files. We observed the average processing times for 1200 images by the

Table 1: Experimental setup and parameters

\begin{tabular}{||l|l||}
\hline Extractors & $\begin{array}{l}4 \text { OpenCV (faces, eyes, closeups, facial } \\
\text { profiles), 1 Tesseract OCR }\end{array}$ \\
\hline $\begin{array}{l}\text { Docker-VM } \\
\text { Image }\end{array}$ & $\begin{array}{l}\text { Ubuntu Trusty (14.04), 8 GB RAM, 4 } \\
\text { vCPU, 10 GB disk space }\end{array}$ \\
\hline Test Images & $\begin{array}{l}\text { Size: 10KB-4MB, PNG format, content: } \\
\text { faces, eyes, closeups, facial profiles, text }\end{array}$ \\
\hline Elasticity Pa- & $\begin{array}{l}\text { Queue threshold: 30 requests, idle timeout } \\
\text { for docker container removal: 10 mins, idle } \\
\text { rameters } \\
\text { timeout for Docker-VM to be suspended: } \\
15 \text { mins, minimum extractor instances re- } \\
\text { quired: 2 }\end{array}$ \\
\hline
\end{tabular}
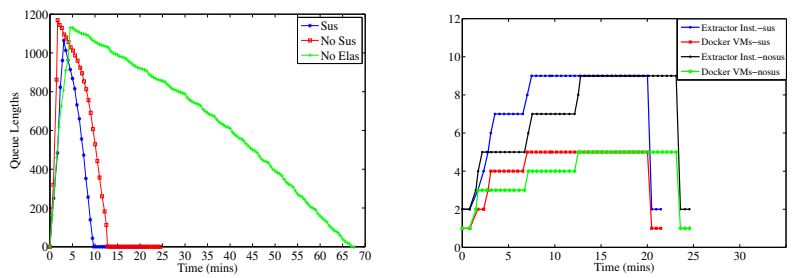

Figure 4: Comparison of queue lengths (left) and the number of extractor instances and number of VMs (right) for the facial profiles extractor, with Scenarios 1, 2 and 3.

five extractors while EM in action. In addition, we also observed the queue lengths and the number of extractor instances and VMs started due to the autoscaling. When scaling up, existing suspended VMs are resumed as opposed to creating a new one to respond faster.

We consider three scenarios for our tests: without elasticity (denoted as Scenario 1), elasticity with no suspended Docker-VMs (Scenario 2), and elasticity with suspended Docker-VMs at the starting of a test (Scenario 3).

Among the five extractors, facial profile extractor is the most CPU-intensive and the OCR and closeup extractors takes less time. Henceforth, we will provide observations for facial profile extractor as its average processing time is affected the most due to autoscaling; and other extractors follow a pattern similar to the facial profile extractor in terms of queue lengths and number of extractor instances/ Docker-VMs. The average processing time for profile was found out to be: 73.25 mins (Scenario 1), 13.26 mins (Scenario 2) and 9.3 mins (Scenario 3). As we see, we have a significant improvement in processing times for the tests when scaling up with EM in Scenarios $2 \& 3$ as compared to no EM in Scenario 1. There are several reasons for this improvements as compared to results obtained from VM image deployment method in [15]: i) The Docker-VMs image has $4 \mathrm{vCPU}$ and can contain upto six containers; ii) launching of a container takes less amount of time in milliseconds as compared to a VM; iii) the containers are spread over different Docker-VMs. This means the facial profiles extractor containers may not be deployed in the same Docker-VM as eyes or faces extractor containers, which have similar time complexity, thus less CPU resource contentions.

The queue length graph in Figure 4 (left) shows the queue lengths over time when the test images are submitted and the time when all are processed for all three scenarios. As expected the time for queue length to become zero in scenario 1 is much more than the other two scenarios. Figure 4 confirms that with EM, the system responded to the increase in load in the system faster than without. The graph to the right shows the reason: creating/resuming Docker-VMs and adding extractor containers on it increased the number of extractor instances. The graph also shows the scaling down behavior. As expected the graphs have shifted left confirming the performance improvement of Docker deployment method over VM image deployment method [15].

\subsection{Scalability within HPC}

To evaluate the first approach of deploying multiple extractor instances as a single job on HPC, we conducted tests with VAT extractors on Pittsburgh Supercomputing Center (PSC) Greenfield, which is an XSEDE resource, where we deployed the Video Analysis (ncsa.cinemetrics) extractor. 
Table 2: VAT Test Results: Video Analysis Extractor

\begin{tabular}{||l|l|l|l|l|l||}
\hline $\begin{array}{l}\text { Internet } \\
\text { Archive } \\
\text { Search } \\
\text { Query }\end{array}$ & $\begin{array}{l}\text { Video } \\
\text { File }\end{array}$ & $\begin{array}{l}\text { Total } \\
\text { File } \\
\text { Size } \\
(\mathrm{MB})\end{array}$ & $\begin{array}{l}\text { Mean } \\
\text { File } \\
\text { Size } \\
(\mathrm{MB})\end{array}$ & $\begin{array}{l}\text { Extractor } \\
\text { Instances } \\
\text { Count }\end{array}$ & $\begin{array}{l}\text { Total } \\
\text { Process- } \\
\text { ing Time } \\
(\mathrm{s})\end{array}$ \\
\hline \hline Insects & 92 & 340.8 & 3.70 & 45 & 1020 \\
\hline Animals & 77 & 736.4 & 9.56 & 45 & 1020 \\
\hline Birds & 34 & 334.4 & 9.83 & 45 & 2040 \\
\hline Tobacco & 72 & 475.8 & 6.60 & 45 & 620 \\
\hline
\end{tabular}

The tests were conducted using videos downloaded from the Internet Archive ${ }^{15}$. We used some common query terms like "animals", "birds" to search the Internet Archive and downloaded video files for each query term. The result set of each search query was then uploaded into separate datasets in Clowder.

Each test consisted of uploading a set of video files into a dataset in Clowder. This triggers processing of those files by the extractor. During each test, multiple instances of the Video Analysis extractor were run inside an HPC job. The HPC batch job contained instructions to run as many extractor instances as there were compute cores requested. So, for example if 45 cores were requested in the job, 45 extractor instances were started. After the jobs were scheduled, we observed the RabbitMQ queue for this extractor. The Video Analysis extractor detects shots in the videos, generates an ensemble of feature descriptors, stores the key frames of the shots as sections of the original video file in the Clowder database. Its computation time is dependent on the size of the videos and the total number of shots detected. The test results are shown in Table 2. Note that in this test the uploaded video files were processed by 45 extractor instances running in parallel.

\section{CONCLUSION AND FUTURE WORK}

In this paper, we described functionalities that have been added to Tools Catalog (TC) and Elasiticity Module (EM). TC allows a user to provide information on any existing data transformation software or new software built by researchers, to upload BD tools/scripts related to those software. The EM was extended to support both VM image and Docker container deployment methods. To automate the deployment of BD tool/script in a timely manner in cloud, TC and EM were integrated. The current implementation of this EM and TC are in a small production status within Brown Dog. From the experimental validation results, the EM performs as expected by scaling up/down the extractors based on user demands and thereby reducing average processing time and resource usages in case an extractor is idle. In future, we will investigate the EM's performance for different data types, file sizes, extractors, and traffic pattern, and will optimize on the data movements within BD services.

We explored two approaches for deployment of extractors on HPC resources. We provided some experimental results on scalability that can be achieved by deploying multiple extractor instances within HPC as a single job. We also provided design modification to extractor to be deployable on HPC.

In the future, we will extend EM to support automatic deployment of extractors on HPC when the queue lengths of

\footnotetext{
${ }^{15}$ archive.org
}

extractors become very large. Also, we will provide options within TC for admin user to choose between different HPC resources or clouds, and provide HPC configuration parameters for deployment of an extractor. We will extend EM so that it can be be used with Amazon cloud service (AWS) and will investigate trade off between cost and performance.

\section{Acknowledgments}

This material is based upon work supported by the National Science Foundation under grant numbers ACI-1053575 (XSEDE) and ACI-1261582 (Brown Dog), and the US Department of Energy, under Award Number DE-AR0000594 (TERRA-REF).

\section{References}

[1] E. Deelman, G. Singh, and et al. Pegasus: A framework for mapping complex scientific workflows onto distributed systems. Scientific Programming Journal, 2005.

[2] M. Dietze, D. LeBauer, and R. Kooper. On improving the communication between models and data. Plant, Cell \& Environment, 2012.

[3] D. Garette and E. Klein. An extensible toolkit for computational semantics. International Conference on Computational Semantincs, 2009.

[4] J. Goecks, A. Nekrutenko, and J. Taylor. Galaxy: A comprehensive approach for supporting accessible, reproducble, and transparent computation research in the life sciences. Genome Biology, 2010.

[5] J. Heard and R. Marciano. A system for scalable visualization of geographic archival records. IEEE Symposium on Large Data Analysis and Visualization, 2011.

[6] J.Towns, T. Cockerill, and et al. XSEDE: Accelerating Scientific Discovery. Computing in Science and Engineering, 16(5):62-74, Sept.-Oct. 2014.

[7] G. Klimeck, M. McLennan, S. Brophy, G. Adams, and M. Lundstrom. nanohub.org: Advancing education and research in nanotechnology. Computing in Science and Engineering, 2008.

[8] V. Kuhn, A. Craig, M. Simeone, S. P. Satheesan, and L. Marini. The vat: Enhanced video analysis. In Proceedings of the 2015 XSEDE Conference: Scientific Advancements Enabled by Enhanced Cyberinfrastructure, XSEDE '15, pages 11:1-11:4, New York, NY, USA, 2015. ACM.

[9] B. Ludascher, I. Altintas, and et al. Scientific workflow management and the kepler system. Concurrence and computation: Practice and Experience, Special Issue on Scientific Workflows, 2006.

[10] L. Marini, R. Kooper, and et al. Medici: A scalable multimedia environment for research. The Microsoft e-Science Workshop, 2010.

[11] K. McHenry, R. Kooper, and P. Bajcsy. Towards a universal, quantifiable, and scalable file format converter. The IEEE Conference on e-Science, 2009.

[12] K. McHenry, R. Kooper, M. Ondrejcek, L. Marini, and P. Bajcsy. A mosaic of software. The IEEE International Conference on eScience, 2011.

[13] W. Michener, S. Allard, and et al. Participatory design of dataone - enabling cyberinfrastruture for the biological and environmental sciences. Ecological Informatics, 2012.

[14] J. Myers, M. Hedstrom, and et al. Towards sustainable curation and preservation: The sead project's data services approach. Interoperable Infrastructures for Interdisciplinary Big Data Sciences Workshop, IEEE eScience, 2015.

[15] S. Padhy, G. Jansen, and et al. Brown dog: Leveraging everything towards autocuration. In IEEE Big Data, 2015.

[16] T. Rath and R. Manmatha. Word spotting for historical documents. International Journal on Document Analysis and Recognition, 2007.

[17] F. Soper. The pronom file format registry. Experts Workgroup on the Preservation of Digital Memory, 2004. 